\title{
Statistical Transmutation in Doped Quantum Dimer Models
}

\author{
C. A. Lamas, ${ }^{1}$ A. Ralko, ${ }^{2}$ D. C. Cabra ${ }^{3}$ D. Poilblanc, ${ }^{1}$ and P. Pujol ${ }^{1}$ \\ ${ }^{1}$ Laboratoire de Physique Théorique, IRSAMC, CNRS and Université de Toulouse, UPS, F-31062 Toulouse, France \\ ${ }^{2}$ Institut Néel, CNRS and Université Joseph Fourier, F-38042 Grenoble, France \\ ${ }^{3}$ IFLP, Departamento de Física, Universidad Nacional de La Plata, La Plata, Argentina
}

(Received 23 February 2012; published 3 July 2012)

\begin{abstract}
We prove a "statistical transmutation" symmetry of doped quantum dimer models on the square, triangular, and kagome lattices: the energy spectrum is invariant under a simultaneous change of statistics (i.e., bosonic into fermionic or vice versa) of the holes and of the signs of all the dimer resonance loops. This exact transformation enables us to define the duality equivalence between doped quantum dimer Hamiltonians and provides the analytic framework to analyze dynamical statistical transmutations. We investigate numerically the doping of the triangular quantum dimer model with special focus on the topological $\mathbb{Z}_{2}$ dimer liquid. Doping leads to four (instead of two for the square lattice) inequivalent families of Hamiltonians. Competition between phase separation, superfluidity, supersolidity, and fermionic phases is investigated in the four families.
\end{abstract}

DOI: 10.1103/PhysRevLett.109.016403

PACS numbers: 71.27.+a, 47.37.+q, 74.25.Ld, 75.10.Kt

The discovery of exotic liquids such as topological $\mathbb{Z}_{2}$ spin liquids $[1,2]$ is one of the challenges of modern condensed matter physics. Anderson proposed that the parent (insulating) state of the high-temperature superconductors is in fact a spin liquid, the resonating valence bond state, and that spin-charge separation (and superconductivity) will occur under doping [3]: the original electron fractionalizes into two emergent particles, a holon carrying the charge quantum and a spinon carrying the spin quantum. Although the original electron is a fermion, there has been a long-standing debate regarding the actual statistics of holons and spinons in such a "deconfinement" scenario. In this context, Rokhsar and Kivelson [4] introduced the quantum dimer model (QDM) as a simple, effective model to describe magnetically disordered phases. The basis assumption here is that the spinon spectrum is gapped [5] (strictly speaking, in the QDM it is infinite), and the dimers between nearest neighbor (NN) sites mimic fluctuating $\mathrm{SU}(2)$ singlets of paired electrons. The underlying microscopic exchange interaction leads to effective attraction or repulsion between dimers and dimer flips along closed loops (see below). Doped QDM, where dimers (i.e., pairs of electrons) are removed from the system, leading to itinerant holes, have also been studied [4,6-8]. There, holes and dimers are strongly coupled due to hard-core constraints. Variants of doped QDM have been also constructed to physically describe polarized spinons induced by a magnetic field [9]. Naively, one expects holons to be of fermionic nature (while spinons should be bosonic), but in spite of this naive expectation, the statistics of holons has been debated over the last 20 years. Earlier work suggested that holon excitations were fermions [10]. It was then argued that, in fact, holon statistics are dictated by energetic considerations, and under some conditions, a holon could become a bosonic composite through binding of a flux quantum [8], the "vison" in the QDM language. This was indeed observed recently by exact diagonalization techniques in the square lattice [7] where, by varying the ratio of dimer kinetic energy vs holon kinetic energy, one can transit from a regime where low-energy quasiparticles behave as fermions to a regime where they behave as bosons.

In this Letter, we prove an exact duality transformation that provides a framework to analyze this dynamical statistical transmutation. More precisely, we study the generic Hamiltonians of QDM's doped with holes depicted in Fig. 1, which contain three contributions: $H_{J}$ and $H_{V}$, the flipping and potential energy terms for the dimers of amplitudes $J$ and $V$, respectively, and $H_{t}$, the hopping term for vacancies (named "holes") of amplitude $t$.

The Hilbert space corresponds to all dimer coverings with a fixed hole density. The potential term is proportional to the number of flippable plaquettes (which is not conserved by dimer flips and hole motion). Special attention is devoted to frustrated lattices, the triangular lattice with edge-sharing triangular units, and the kagome lattice with corner-sharing triangular units, which we compare to the results obtained for the square lattice [7]. The exact symmetry of these Hamiltonians states that their spectrum is invariant under simultaneous transformations of the statistics of the holes, bosons into fermions or vice versa, $\mathcal{B} \leftrightarrow \mathcal{F}$, and of the sign of (all) dimer kinetic amplitudes, $J \leftrightarrow-J, V$ and $t$ being unchanged.

Equivalence classes.-For fixed values of the magnitudes of $J, V$, and $t$, one can define eight different Hamiltonians by changing the signs of $J$ and/or $t$ and by choosing bosonic or fermionic statistics for the mobile holes. Using the exact symmetry mentioned above and proven below, these eight different Hamiltonians can be grouped in pairs with identical spectra, hence 


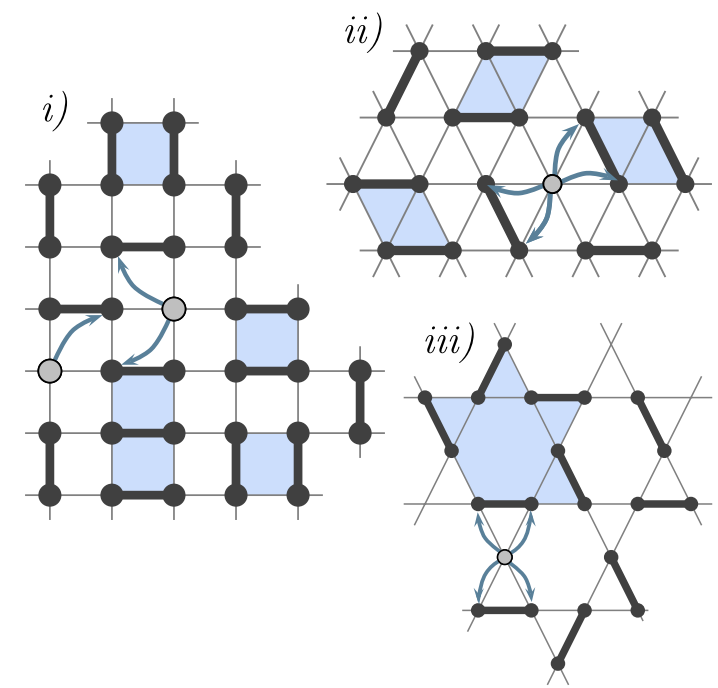

FIG. 1 (color online). Dimer coverings corresponding to different lattices. Light blue shaded areas represent flippable plaquettes and arrows represent the allowed single hole hopping process. (i) Square lattice: The hole hopping is between sites in the same sublattice. (ii) Triangular lattice: A hole can hop in the different directions schematized by the arrows. (iii) Kagome lattice: The light blue shaded area shows one of the possible flippable plaquettes of length 10 .

defining four nonequivalent families. This is summarized in Fig. 2.

More precisely, the established equivalence is between $H_{1}$ and $H_{2}$, both represented by the family label (a) and in the same way for the pairs of Hamiltonians $H_{2 i-1}$ and $H_{2 i}$ with $i=2,3,4$ corresponding, respectively, to families (b), (c), and (d). We argue that such an equivalence is a general feature of doped two-dimensional QDMs, (a)

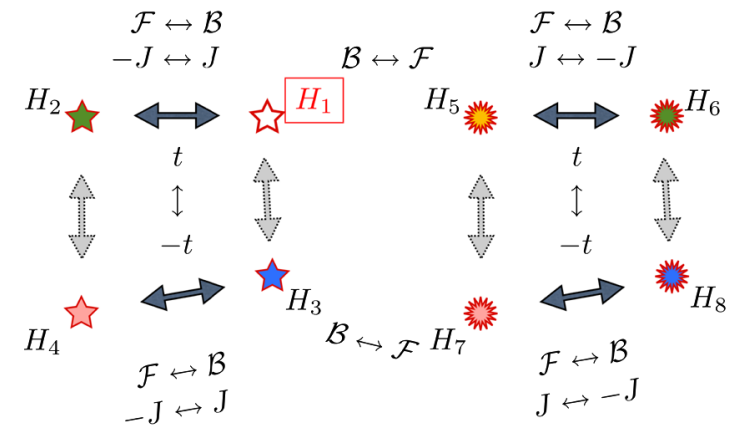

doped “spinonic” QDM doped "electronic" QDM
FIG. 2 (color online). Equivalence classes ("models") and relations between the eight Hamiltonians introduced in the text. Starting from the unfrustrated $(J>0, t>0)$ bosonic $H_{1}$ Hamiltonian, one can combine any change of statistics and/or change of signs of $J$ and/or sign of $t$ to obtain $H_{2}, \cdots, H_{8}$. Equivalence relations are shown by arrows: full dark grey arrows (dotted light grey arrows) are valid for all lattices (for vacancies hopping on the square lattice only). independent of the lattice geometry. Physically, classes (a) and (b) [(c) and (d)] describe systems with doped polarized spinons (doped holons) and are called "spinonic" QDMs ("electronic" QDMs). Note that, for the square (or hexagonal) lattice, an additional $t \leftrightarrow-t$ symmetry reduces the number of nonequivalent families from 4 to 2. In Fig. 2, the two families defined for the square lattice by $H_{1} \leftrightarrow H_{2} \leftrightarrow H_{3} \leftrightarrow H_{4}$ on one hand and by $H_{5} \leftrightarrow$ $H_{6} \leftrightarrow H_{7} \leftrightarrow H_{8}$ on the other hand, were dubbed as "Perron-Frobenius" and "non-Perron-Frobenius" Hamiltonians and studied in [7], while for the triangular lattice, only the unfrustrated $H_{1}$ Hamiltonian [representing family (a)] was studied in [9].

As the transformation we shall establish below change bosons into fermions (and vice versa), we can start by assuming, without lost of generality, that the (bare) holons in the doped QDM are bosons. We implement a twodimensional Jordan-Wigner transformation on these bosons to change their statistics to fermionic. In contrast to one-dimensional systems, this resulting transformed Hamiltonian is highly nonlocal [11-14], requiring, in general, a mean field approximation to proceed further, at least from an analytical point of view. Next, we show that the nonlocal terms can be absorbed by using a different representation for the dimer operators, which keeps their bosonic character. This key feature provides then an elegant proof of the "statistical transmutation symmetry" of these models.

Proof of statistical transmutation symmetry.-It is convenient to write the Hamiltonian in a second quantized form by introducing creation operators $b_{i, j}^{\dagger}$ for a dimer sitting between sites $i$ and $j$ and holes by operators $a_{i}^{\dagger}$. In our conventions, dimers between sites $i$ and $j$ are created by spatially symmetric operators $b_{i, j}^{\dagger}$; both operators $\left(b_{i, j}^{\dagger}\right.$ and $a_{i}^{\dagger}$ ) are bosonic and mutually commuting. It is instructive to notice that the dimer bosonic operators can be thought of as bilinears of "electrons" operators: $b_{i, j}^{\dagger}=$ $\frac{1}{\sqrt{2}}\left(c_{i, \uparrow}^{\dagger} c_{j, \downarrow}^{\dagger}-c_{i, \downarrow}^{\dagger} c_{j, \uparrow}^{\dagger}\right)$. In terms of these operators, we implement the hard-core constraint $a_{i}^{\dagger} a_{i}+\sum_{z} b_{i, i+z}^{\dagger} b_{i, i+z}=1$, where the sum runs over the NN of site $i$. Let us call $\hat{\mathcal{P}}$ the projector on the subspace where the constraint holds. In the following, we use systematically the projected Hamiltonian $\hat{\mathcal{P}} H \hat{\mathcal{P}}(=H \hat{\mathcal{P}}$ since all terms of $H$ commute with $\hat{\mathcal{P}}$ ), which proves to be very useful later.

Let us now apply a 2D Jordan-Wigner (JW) transformation on the holon operators [12]:

$$
a_{i}=e^{-i \phi_{i}} f_{i}
$$

where $\phi_{i}=\sum_{j \neq i} f_{j}^{\dagger} f_{j} \arg \left(\tau_{j}-\tau_{i}\right)$, and $\tau_{j}=x_{j}+i y_{j}$ is the complex coordinate of the $j$ th hole. Using that $\arg \left(\tau_{i}-\tau_{j}\right)=\arg \left(\tau_{j}-\tau_{i}\right) \pm \pi$, it follows immediately that two $f$ operators in different sites anticommute, and 
the constraint and the phase $\phi_{i}$ can be written equally in terms of $f_{i}$ or $a_{i}$ operators.

In order to understand the consequences of transformation (1) on the Hamiltonian, the hopping of holons can be written, for an arbitrary lattice, as a sum of three-site Hamiltonians,

$$
H_{t}=\sum h_{(i j k)}^{(t)} \quad \text { with } \quad h_{(i j k)}^{(t)}=t \hat{\mathcal{P}} b_{i, j}^{\dagger} b_{j, k} a_{k}^{\dagger} a_{i} \hat{\mathcal{P}} .
$$

Making use of transformation (1) we obtain

$$
h_{(i j k)}^{(t)}=t e^{i \arg \left(\tau_{k}-\tau_{i}\right)} \hat{\mathcal{P}} e^{i \phi_{k}} e^{-i \phi_{i}} b_{i, j}^{\dagger} b_{j, k} f_{k}^{\dagger} f_{i} \hat{\mathcal{P}} .
$$

In the last equation, we have changed boson operators $a_{i}$ to fermionic ones $f_{i}$ at the cost of introducing nonlocal interactions. In other words, we have written a boson as a composite particle consisting of an electron with an attached flux. As we show just below, this nonlocal interaction can be absorbed in a redefinition of the dimer operators without affecting their bosonic commutations relation. In order to define new dimer operators including the phases $\phi$ in their definition, we must be able to write $\phi$ in terms of operators $b_{i, j}$. This can be performed in the following way: when applied into the subspace projected by $\hat{\mathcal{P}}$, we can change $\phi$ by $\tilde{\phi}$ in the exponentials, where

$$
\tilde{\phi}_{i}=\sum_{r \neq i}\left[1-\sum_{z} b_{r, r+z}^{\dagger} b_{r, r+z}\right] \arg \left(\tau_{r}-\tau_{i}\right) .
$$

The remaining nonlocal exponential operators are rewritten in terms of $\tilde{\phi}$ which can be absorbed by defining $\tilde{b}_{i, j}=e^{i\left(\tilde{\phi}_{i}+\tilde{\phi}_{j}\right)} b_{i, j}$. As shown above, it is a simple matter to see that operators $\tilde{b}_{i, j}$ are also bosonic, as they should be to make sense as dimer operators. Then, transformation (1) together with the definition of $\tilde{b}_{i, j}$ allow us to change the statistics of holes from bosonic to fermionic. After this transformation, the hopping term is written in terms of operators $\tilde{b}_{i, j}$ and $f_{i}$, and the hopping amplitude changes to $\tilde{t}=t e^{i\left[\pi+\arg \left(\tau_{j}-\tau_{i}\right)-\arg \left(\tau_{j}-\tau_{k}\right)\right]}$. Let us now investigate the effect of this transformation specifically for each lattice.

(i) Square lattice.-The Hamiltonian is defined by $H_{1}=H_{J}+H_{V}+H_{t}$, where

$$
\begin{aligned}
& H_{J}=-J \sum_{\square}\{|\mathfrak{l} \mathfrak{l}\rangle\langle\because \bullet|+\text { H.c. }\}
\end{aligned}
$$

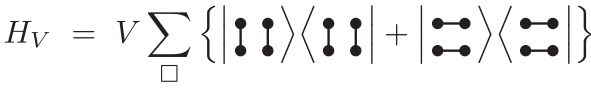

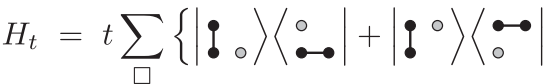

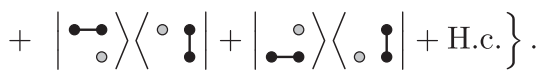

Before going into the details of the Jordan-Wigner transformed Hamiltonian, let us mention some well-known gauge transformations which can be performed. For zero doping, a simple gauge transformation on the dimers can be done to show the equivalence of the Hamiltonians with $J$ and $-J$. In the case of nonzero doping, changing the sign of the holes wave function with a wave vector transformed as $\vec{k} \rightarrow \vec{k}+(\pi, \pi)$ one obtains easily the equivalence between Hamiltonians with $t$ and $-t$. We can now proceed to the computation of the Jordan-Wigner transformed Hamiltonian. The transformed Hamiltonian becomes, after some algebra [see Supplemental Material (SM) [15]], $H_{2}=\tilde{H}_{J \rightarrow-J}+\tilde{H}_{V}+\tilde{H}_{t}$, where the tilde means that dimers and holes are created by operators $\tilde{b}^{\dagger}$ and $f^{\dagger}$ respectively. In the dimer kinetic Hamiltonian, the amplitude $J$ has been changed by $-J$.

(ii) Triangular lattice.-Here, the Hamiltonian $H_{1}$ is written using

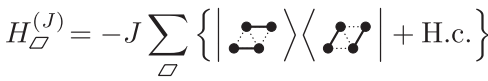

$$
\begin{aligned}
& H_{\square}^{(V)}=V \sum_{\square}\{|\because\rangle\langle\because|+| \mathfrak{\bullet}\rangle\langle\mathscr{\bullet}|\}
\end{aligned}
$$

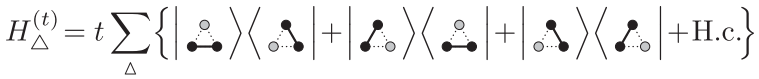

and similar expressions for

$$
H_{\square}
$$

and

$$
H_{\diamond}
$$

for the other orientations of the rhombi [16]. $H_{\Delta}^{(t)}$ corresponds to the holon hopping on the up triangles, the total hopping Hamiltonian being $H_{t}=H_{\triangle}^{(t)}+H_{\nabla}^{(t)}$. It is important to note that on such nonbipartite lattices the equivalence $t \leftrightarrow-t$ does not hold anymore, as verified numerically. This implies that there are now four families of equivalent Hamiltonians, instead of two for the square case. On the triangular lattice, the dual Hamiltonian reads

$$
H_{2}=\tilde{H}_{\square}^{(J) \rightarrow(-J)}+\tilde{H}_{\square}^{(V)}{ }^{\prime}+\tilde{H}^{(t)},
$$

with the same convention as before (see SM [15]). A corollary of this result is that, as for the square lattice, the Hamiltonians with $J$ and $-J$ are equivalent in the zero doping case.

(iii) Kagome lattice.-On this geometry, all dimer resonant loops (of length $\alpha=6,8,10,12$ ) involving a single hexagon become important and should be considered [17]. Nearest-neighbor hole hopping as depicted in Fig. 1 can be introduced as in [18] (the reader can refer to [17,18] for more details). The JW transformed Hamiltonian is obtained in the same way as before, and since it is interesting on its own, it will be presented elsewhere [19]. Here, we only summarize the main results. By using the JW transformation followed by (more involved) gauge transformations on dimer and hole operators, [20] one can write the dual Hamiltonian with all kinetic dimer amplitudes $J_{\alpha}$ changed to $-J_{\alpha}$. As before, dimers and holons are created by $\tilde{b}^{\dagger}$ and $f^{\dagger}$, respectively. 
Statistical transmutation and choice of Hamiltonian.The above statistical transmutation symmetry is of great importance in analytic and numerical investigations of doped QDMs, establishing their phase diagrams. In [7], it was shown that in the square lattice, by varying the ratio $J / t$ or the doping, the system undergoes a series of phase transitions. One of those phase transitions corresponds to a dynamical transmutation between a phase where elementary low-energy quasiparticles are fermionic holes to a phase where the low-energy quasiparticles acquire a bosonic nature.

It was in fact predicted that holon and vison could pair up, leading to such statistical transmutation [8]. Then, if the microscopic Hamiltonain is chosen such that holons are fermions, the fermionic phase corresponds to a weakcoupling regime and the bosonic phase to a strongcoupling one. This picture gets interchanged if holons in the microscopic Hamiltonian are chosen to be bosons. Hence, using this duality equivalence one can always choose the most relevant microscopic QDM Hamiltonian to be in a weak-coupling regime (depending on the point in the phase diagram).

Phase diagrams. - We now complement the exact results with a numerical study of the phase diagram of the four nonequivalent families of Hamiltonians in the triangular lattice where a topological $\left(\mathbb{Z}_{2}\right)$ liquid can be stabilized at zero doping $[2,21]$. The statistics of the dressed excitations is studied using the method developed in [7] by investigating the node content of the wave functions. Family (a) in Fig. 3 is the only unfrustrated case and was studied with

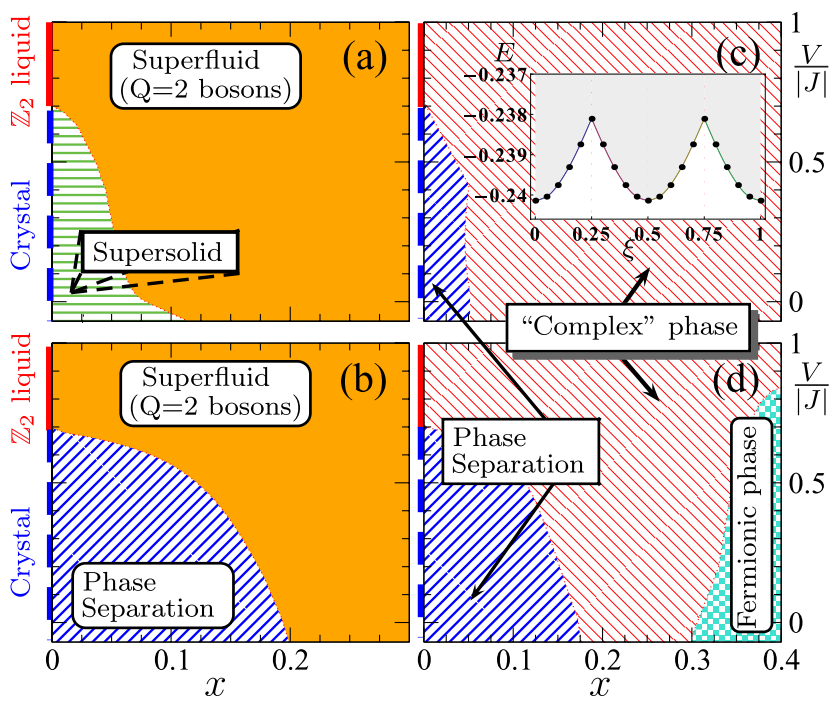

FIG. 3 (color online). Qualitative phase diagrams of the four families of doped QDM's (a)-(d) as labeled in Fig. 2 on the triangular lattice versus doping $(x)$ and $V /|J|$ for fixed $t / J=0.5$. Inset: Energy vs normalized magnetic flux $(\xi)$ corresponding to family (c) for fixed $V /|J|=0.5, t / J=0.5$ and $x=0.25$. The $E$ vs $\xi$ plots corresponding to families (a), (b), and (d) are qualitatively similar.
Green function Monte Carlo methods in [9]. In this model, bare holons are bosonic and remain representative of the physical excitations in the entire region of the phase diagram, as happen also in the Perron-Frobenius square lattice version studied in [7]. The unfrustrated model shows a superfluid phase in a wide range of the phase diagram.

The situation is even more interesting when $J$ is changed to $-J$ or, equivalently, bosons are changed into fermions [families (c) and (d) in Fig. 3]. Most of the phase diagram is occupied by what we dubbed a "complex phase" as the statistics of dressed excitations do not correspond solely to bosons or fermions. Similarly to the square lattice, we find a phase separated region at low doping. Family (d) also shows, in addition, an interesting fermion reconstruction at large doping, which we call "fermionic phase".

In order to provide a better understanding of the conducting phases of the triangular lattice, we insert in the torus an Aharonov-Bohm flux of strength $\phi=\xi \phi_{0}$ with $0 \leq \xi \leq 1$ (achieved by adding a phase shift in, e.g., the $x$ direction) and where $\phi_{0}=h c / e$ the elementary magnetic flux. As done in Ref. [6] on the square lattice, one can reduce the finite-size effects by considering arbitrary boundary conditions in the $y$ direction. A superfluid is characterized by well-defined minima in the ground-state energy separated by a finite barrier in the thermodynamic limit. On the contrary, a typical signature of (weakly interacting) fermions, a flat energy profile, is expected even on such a small cluster [22]. Here, we report that the ground-state energy has well-defined minima quantized at half a flux quantum for all families of models at $x \sim 0.25$, compatible with a bare charge $Q=2 e$ superconductor [6,23] [see inset of Fig. 3(c)]. Further detailed discussions on the charge of the "low-energy" particles in the superconducting phase will be provided elsewhere [19].

Discussion and perspectives.-In this Letter, we have shown that the nature of the excitations in doped QDM is a much more subtle question than what one would naively think. We have rigorously established equivalence classes between QDM Hamiltonians (see Fig. 2). We claim that this duality relation is a generic feature of QDM, independent of the details of the lattice.

This proven duality relation provides a powerful tool to identify the nature of the dressed hole excitations. Indeed, imagine one considers a specific system with given microscopic parameters corresponding to one of the four (or two) families of Hamiltonians. A priori, two (or four) representations of this system can be chosen freely using the equivalence relations of Fig. 2. As we have shown, there is in fact a specific representation which corresponds to a weakly coupled regime, i.e., where the chosen statistic of the bare holes is the one of the true dressed excitations. This is the representation to be chosen to study, e.g., the effect of further perturbations of the system.

Lastly, we have shown numerically that models on the triangular lattice corresponding physically to spinon or 
holon doping have very different phase diagrams with either a superfluid or a "complex" phase (in which dressed excitations cannot be understood solely in terms of fermionic or bosonic degrees of freedom), respectively. In the latter case, we provide evidence of statistical transmutation, analogous to the case of the square lattice.

A.R., D.P., and P.P. acknowledge support by the Agence Nationale de la Recherche under Grant No. ANR 2010 BLANC 0406-0. C. A. L. and D. C.C. are partially supported by CONICET (PIP 1691) and ANPCyT (PICT 1426).

[1] X.-G. Wen, Adv. Phys. 44, 405 (1995), and references therein.

[2] R. Moessner and S. L. Sondhi, Phys. Rev. Lett. 86, 1881 (2001).

[3] P.W. Anderson, Science 235, 1196 (1987); P. W. Anderson, Mater. Res. Bull. 8, 153 (1973); P. Fazekas and P. W. Anderson, Philos. Mag. 30, 423 (1974).

[4] D. S. Rokhsar and S. A. Kivelson, Phys. Rev. Lett. 61, 2376 (1988).

[5] N. Read and S. Sachdev, Phys. Rev. Lett. 66, 1773 (1991).

[6] A. Ralko, F. Mila, and D. Poilblanc, Phys. Rev. Lett. 99, 127202 (2007); see also D. Poilblanc, F. Alet, F. Becca, A. Ralko, F. Trousselet, and F. Mila, Phys. Rev. B 74, 014437 (2006).

[7] D. Poilblanc, Phys. Rev. Lett. 100, 157206 (2008).

[8] S. Kivelson, Phys. Rev. B 39, 259 (1989).
[9] A. Ralko, F. Becca, and D. Poilblanc, Phys. Rev. Lett. 101, 117204 (2008).

[10] N. Read and B. Chakraborty, Phys. Rev. B 40, 7133 (1989).

[11] E. Fradkin, Phys. Rev. Lett. 63, 322 (1989).

[12] Y. R. Wang, Phys. Rev. B 43, 3786 (1991).

[13] D. C. Cabra and G. L. Rossini, Phys. Rev. B 69, 184425 (2004).

[14] C. A. Lamas, D. C. Cabra, M. D. Grynberg, and G. L. Rossini, Phys. Rev. B 74, 224435 (2006).

[15] See Supplemental Material at http://link.aps.org/ supplemental/10.1103/PhysRevLett.109.016403 for the technical details.

[16] A. F. Albuquerque, H. G. Katzgraber, M. Troyer, and G. Blatter, Phys. Rev. B 78, 014503 (2008).

[17] D. Poilblanc, M. Mambrini, and D. Schwandt, Phys. Rev. B 81, 180402(R) (2010).

[18] D. Poilblanc and A. Ralko, Phys. Rev. B 82, 174424 (2010).

[19] C. A. Lamas, A. Ralko, D. C. Cabra, D. Poilblanc, M. Oshikawa, and P. Pujol (unpublished).

[20] The gauge transformation allows us to bring the hopping term back to its original form after changes in the hopping amplitudes generated by the statistical transmutation. For convenience, details will be published in [19].

[21] A. Ralko, M. Ferrero, F. Becca, D. Ivanov, and F. Mila, Phys. Rev. B 71, 224109 (2005).

[22] D. Poilblanc, Phys. Rev. B 44, 9562 (1991).

[23] S. A. Kivelson, D. S. Rokhsar, and J. P. Sethna, Europhys. Lett. 6, 353 (1988). 